Critique de la santé publique. Une approche anthropologique. Jean-Pierre Dozon et Didier Fassin (Dir.)

Paris, Balland (coll. Voix et regards), 2001, ISBN 2715813767, 362 p.

\title{
Marc-Eric Gruénais
}

\section{(2) OpenEdition}

\section{Journals}

Édition électronique

URL : http://journals.openedition.org/apad/192

DOI : 10.4000/apad.192

ISSN : 1950-6929

Éditeur

LIT Verlag

Édition imprimée

Date de publication : 1 juin 2001

\section{Référence électronique}

Marc-Eric Gruénais, «Critique de la santé publique. Une approche anthropologique. Jean-Pierre Dozon et Didier Fassin (Dir.) », Bulletin de l'APAD [En ligne], 21 | 2001, mis en ligne le 06 mars 2006, consulté le 23 septembre 2020. URL : http://journals.openedition.org/apad/192 ; DOI : https://doi.org/10.4000/ apad.192

Ce document a été généré automatiquement le 23 septembre 2020.

Bulletin de l'APAD 


\title{
Critique de la santé publique. Une approche anthropologique. Jean-Pierre Dozon et Didier Fassin (Dir.)
}

Paris, Balland (coll. Voix et regards), 2001, ISBN 2715813767, 362 p.

\author{
Marc-Eric Gruénais
}

1 La médecine, à l'évidence, a toujours été productrice de normes. La prise en compte des déterminants de santé, aujourd'hui à partir de l'épidémiologie, par des approches qualifiées de "santé publique", pour améliorer l'état de santé des populations se situe, par définition, dans cette perspective normative. Comme le montre très bien $\mathrm{H}$. Hudebine en comparant les positions françaises et anglaises à l'égard de la toxicomanie, les approches de santé publique peuvent être multiples. Toujours à propos de la toxicomanie, A. Lovel, analysant le jeu complexe des pratiques des usagers de drogue, illustre les tentatives d'une mise en forme et en pratique d'une réflexion orientée vers la "réduction des dommages" (pour l'usager de drogue) qui tente de s'éloigner de la normativité inhérente à l'approche risque. Les mesures de santé publique s'inscrivent toujours, peu ou prou, au sein d'une tension entre un modèle libéral valorisant la responsabilité individuelle et une gestion autoritaire holiste de contrôle des individus, comme le souligne M. Calvez. Néanmoins, toute tentative de généralisation ne doit pas faire oublier l'existence de positionnement différents de santé publique selon le type de problème de santé envisagé (infection par le VIH, toxicomanie), selon le pays, mais aussi selon l'époque. Les articles de L. Berlivet et de J.P. Gaudillère apportent ici des éclairages utiles sur l'évolution de la notion de risque dans l'histoire de l'épidémiologie depuis la fin du XIXe siècle, pour le premier, et sur les décalages successifs de la notion de causalité, pour le second, depuis le risque mis en rapport avec des prédispositions familiales jusqu'à la "génétisation" des étiologies et à la médecine prédictive aujourd'hui. Cette diversité, dans le temps et dans l'espace, J.P. Dozon tente de la systématiser en identifiant quatre modèles de prévention: modèle 
magico-religieux, modèle de la contrainte profane, modèle pastorien, et modèle contractuel, ces différents modèles n'étant pas exclusifs les uns des autres.

2 A ces raffinements successifs en rapport avec la notion centrale de risque, à des approches de santé publique complexes et hétérogènes semblent venir s'opposer des généralisations massives, voire hâtives dès lors que l'on s'intéresse aux populations du Sud. C'est ce que montre bien l'article de L. Vidal à propos de la transmission mère-enfant du VIH en Côte d'Ivoire. Alors que le savoir épidémiologique crée de l'incertitude, sont proposés des discours simplificateurs, voire simplistes, à destination des personnes exposées et pour lesquelles les discours techniques ne répondent pas aux demandes. Cette tendance à la simplification, on la retrouve aussi dans les travaux de Tobie Nathan que critique J. Andoche, qui souligne notamment combien T. Nathan s'est éloigné de la perspective de l'ethnopsychiatrie complémentariste de Devereux dont il se réclame, et apparaît bien davantage comme un "médiateur ethno-clinicien" dont l'intervention est guidée par des présupposés globalisant sur la position de l'immigré face à sa culture. La "culturalisation" des problèmes de santé, lorsqu'il s'agit de parler des sociétés différentes, équivaut, pour D. Passin, à la négation du statut politique de l'Autre. Et face à un problème comme la drépanocytose, qui affecte de manière privilégiée les populations noires et donc qui prête facilement le flanc aux interprétations culturalistes, D. Bonnet propose de développer un "conseil génétique" non fondé sur les considérations ethniques. Dès lors, s'agissant par exemple de la progression de l'infection par le VIH en Afrique, les explications culturelles, voire même sociologiques sont insuffisantes: "ni les habitus culturels, ni le multi-partenariat, ni la condition de dépendance des femmes, ni la pauvreté en tant que telle, ni même la possible sous-estimation de la transmission sanguine ou des déficits nutritionnels, ne sauraient suffire, pris ensemble ou isolément, à expliquer semblables évolutions", écrit J.P. Dozon. Il poursuit en faisant remarquer que les débats sur l'accessibilité des antirétroviraux dans les pays du Sud obligent désormais à considérer d'autres moyens de lutte que la prévention et l'information, et invitent à s'interroger sur les questions d'organisation.

La conclusion de J.P. Dozon et D. Passin fait écho à un des articles de D.Passin qui figure dans la première partie de l'ouvrage qui considère que la santé publique se situe constamment entre utopie et idéologie et est gouvernée par des choix moraux. Au terme du livre, les éditeurs proposent notamment de dépasser le dilemme d'une santé publique tiraillée entre la production de normes et la prise en compte des exceptions culturelles grâce à une anthropologie de la santé qui s'interroge sur les conditions effectives d'un droit à la santé. Lourde tâche à laquelle devra se livrer l'anthropologie sociale, surtout face à la diversité des problèmes et des approches soulignée par les articles de cet ouvrage et dont le titre, "Critique de la santé publique", qui pourrait faire croire à l'existence d'une discipline unifiée, rend mal compte. 